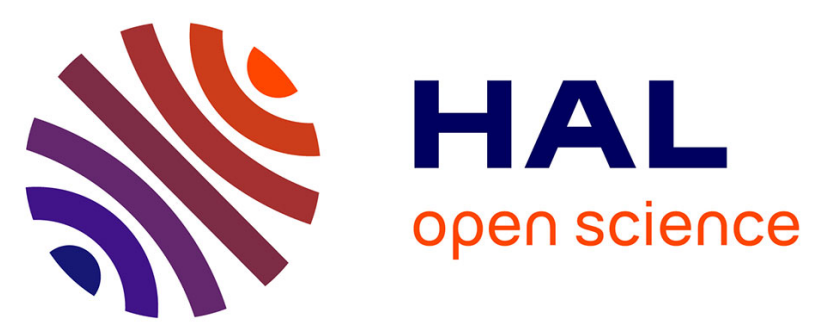

\title{
Analysis of laser speckle contrast images variability using a novel empirical mode decomposition: comparison of results with laser doppler flowmetry signals variability
}

Anne Humeau-Heurtier, Pierre Abraham, Guillaume Mahé

\section{- To cite this version:}

Anne Humeau-Heurtier, Pierre Abraham, Guillaume Mahé. Analysis of laser speckle contrast images variability using a novel empirical mode decomposition: comparison of results with laser doppler flowmetry signals variability. IEEE Transactions on Medical Imaging, 2015, 34 (2), pp.618-627. 10.1109/TMI.2014.2364079 . hal-01164083

HAL Id: hal-01164083

https://hal-univ-rennes1.archives-ouvertes.fr/hal-01164083

Submitted on 16 Jun 2015

HAL is a multi-disciplinary open access archive for the deposit and dissemination of scientific research documents, whether they are published or not. The documents may come from teaching and research institutions in France or abroad, or from public or private research centers.
L'archive ouverte pluridisciplinaire HAL, est destinée au dépôt et à la diffusion de documents scientifiques de niveau recherche, publiés ou non, émanant des établissements d'enseignement et de recherche français ou étrangers, des laboratoires publics ou privés. 


\title{
Analysis of laser speckle contrast images variability using a novel empirical mode decomposition: comparison of results with laser Doppler flowmetry signals variability
}

\author{
Anne Humeau-Heurtier, Pierre Abraham, and Guillaume Mahé
}

\begin{abstract}
Laser Doppler flowmetry (LDF) and laser speckle contrast imaging (LSCI) have emerged as non invasive optical modalities to monitor microvascular blood flow. Many studies proposed to extract physiological information from LDF by analyzing signals variability. By opposition, such analyses for LSCI data have not been conducted yet. We propose to analyze LSCI variability using a novel data-driven method: the complete ensemble empirical mode decomposition with adaptive noise (CEEMDAN). CEEMDAN is suitable for non linear and non stationary data and leads to intrinsic mode functions (IMFs). It is based on the ensemble empirical mode decomposition (EEMD) which relies on empirical mode decomposition (EMD). In our work the average frequencies of LSCI IMFs given by CEEMDAN are compared with the ones given by EMD and EEMD. Moreover, LDF signals acquired simultaneously to LSCI data are also processed with CEEMDAN, EMD and EEMD. We show that the average frequencies of IMFs given by CEEMDAN depend on the signal to noise ratio (SNR) used in the computation but, for a given SNR, the average frequencies found for LSCI are close to the ones obtained for LDF. By opposition, EEMD leads to IMFs with frequencies that do not vary much when the SNR level is higher than a threshold. The new CEEMDAN algorithm has the advantage of achieving a complete decomposition with no error in the reconstruction but our study suggests that further work is needed to gain knowledge in the adjustment of the added noise level. CEEMDAN, EMD and EEMD are data-driven methods that can provide a better knowledge of LSCI.
\end{abstract}

Index Terms-Laser speckle contrast imaging, Empirical mode decomposition, Biomedical image processing, Laser Doppler flowmetry, Microvascular blood flow.

\section{INTRODUCTION}

$\mathbf{T}$ HE monitoring, modeling and processing of microvascular blood flow data is of clinical interest for early diagnosis of pathologies or as surrogate markers (see, e.g., [1], [2], [3]). Thus, for pathologies as diabetes or hypertension early microvascular changes have been shown to appear long before organ dysfunctions become clinically manifest (see,

A. Humeau-Heurtier is with University of Angers, LARIS - Laboratoire Angevin de Recherche en Ingénierie des Systèmes, 62 avenue Notre-Dame du Lac, 49000 Angers, France e-mail: anne.humeau@univ-angers.fr

P. Abraham is with University of Angers, CHU d'Angers, Laboratoire de Physiologie et d'Explorations Vasculaires, UMR CNRS 6214-INSERM 1083, 49033 Angers cedex 01, France.

G. Mahé is with University of Rennes 1, CHU de Rennes, Pôle imagerie médicale et explorations fonctionnelles, INSERM CIC 1414, 35033 Rennes Cedex 9, France.

Manuscript received 4 June, 2014 e.g., [4], [5]). For systemic sclerosis, burns, flaps, or wounds, skin microvasculature is specifically affected and evoluates with the disease ([6], [7]). Different optical techniques have become available to monitor microvascular blood flow. Among them, the laser Doppler flowmetry (LDF) and the laser speckle contrast imaging (LSCI) offer advantages for a continuous and non invasive monitoring of microvascular blood flow ([8], [9]). LDF was introduced in the 1970s ([10]) and is now a commonly used technique that provides an index of perfusion, see an example in Fig. 1. The principle relies on the analysis of the Doppler shifts that are induced by the interactions between photons of a laser light and moving blood cells of the microcirculation in the tissue under study: in LDF the perfusion is proportional to the integral of the frequency-weighted Doppler power spectrum of the backscattered photons and can be written in the form

$$
\text { Perfusion }_{\mathrm{LDF}} \sim \int_{0}^{\infty} \omega P(\omega) d \omega
$$

where $P(\omega)$ is the power spectrum for only the frequencyshifted part of the light (i.e. $P(\omega)=0$ when $\omega=0$ ) ([11]). LDF has the advantage of having a well-established theory ([12]). However, it is a single-point monitoring technique with low reproducibility ([13], [14]). Based on the LDF principle, laser Doppler imagers have been proposed to obtain 2D perfusion images but they rely on a scan of the zone under study (see, e.g., [15], [16]). The acquisition is therefore low, and rapid physiological phenomena may be missed with such imagers. Full-field laser Doppler imagers have been proposed in the last years but necessitate high-speed cameras ([17], [18], [19], [20], [21], [22], [23], [24]). Very recently, laser speckle contrast imagers have been commercialized and have the advantage of being full-field and based on low cost devices, compared to other techniques ([25], [26]). As for LDF, LSCI relies on the interaction of a laser light with the tissue under study. In LSCI the scattered light is imaged onto a camera. Due to constructive and destructive interferences coming from phase differences involved in the backscattered light, speckle patterns are obtained on the camera. Moreover, the movements of the scattering particles in the analyzed tissue generate dynamic speckled images. The exposure time of the camera leads to the integration of the intensity variations which results in a blurring of the speckle pattern. The degree of blurring is 


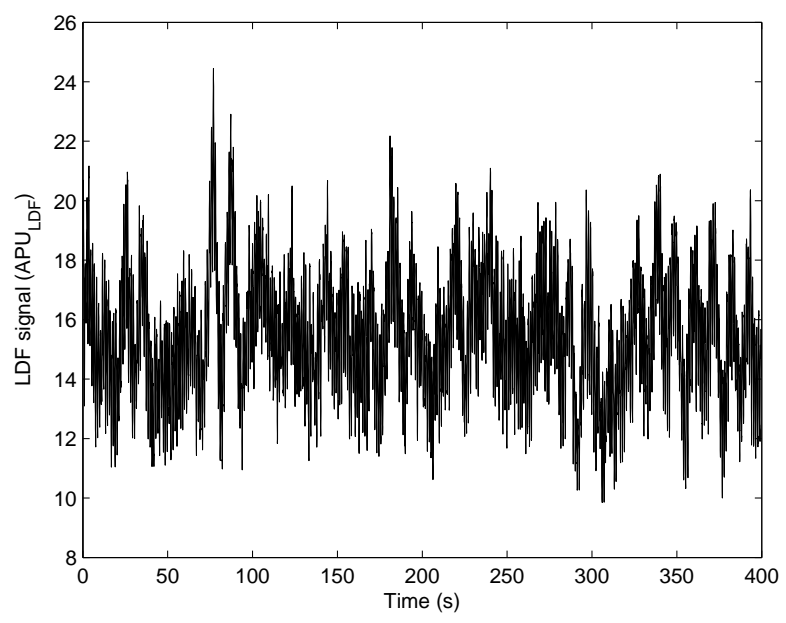

Fig. 1. Laser Doppler flowmetry signal recorded on the forearm of a healthy subject at rest.

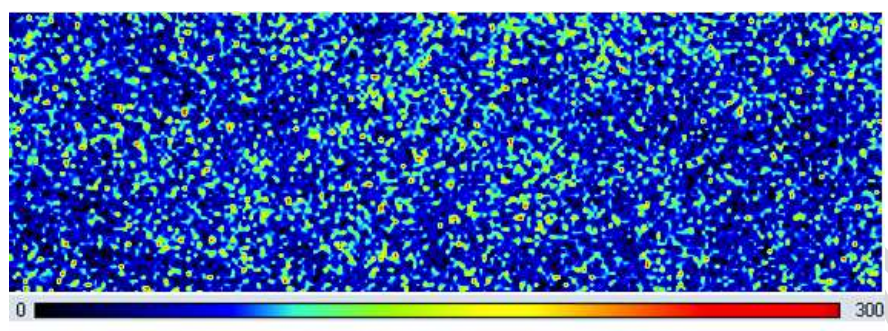

Fig. 2. Laser speckle contrast image of a zone on the forearm of a healthy subject.

given by the speckle contrast $K$ that is computed as

$$
K(x, y)=\frac{\sigma_{N}}{\mu_{N}}
$$

where $\sigma_{N}$ and $\mu_{N}$ are respectively the standard deviation and mean of the pixel intensity in a neighborhood around the pixel in the speckle raw data. The LSCI perfusion index is then computed from the contrast values: LSCI perfusion value is inversely proportional to the contrast $K$, see an example in Fig. 2. Many works on instrumentation and processing of LSCI data are still proposed ([26], [27], [28], [29]). Even if LSCI has the advantage of giving images with high temporal and spatial resolutions, LSCI data are not fully understood yet. In particular, the physiological activities that can be extracted from the images are still unknown.

From biomedical data, physiological activities can be extracted through spectral analyses (see, e.g., [30], [31], [32]). LDF signals have thus been the subject of several spectral analyses ([33]). Two commonly used methods for spectral studies are the (fast) Fourier transform and autoregressive models (see, e.g., [34]). However, with these methods, implicit assumptions of linearity and stationarity are required. Nevertheless, such assumptions are not valid for biomedical data. Wavelet decompositions have also been extensively applied to extract physiological activities (see, e.g., [35] for LDF signals). Wavelet decompositions are standard kernel based approaches. Empirical mode decomposition (EMD) has been introduced at the end of the 1990s as a fully data-adaptive method to extract fast and slow oscillations of non linear and non stationary signals ([36], [37]). From that time, EMD has been used in many biomedical studies for diagnoses purposes (see, e.g., [38]). However, EMD has some drawbacks (presence of "mode mixing", see below). Therefore, a new EMD-based algorithm, the ensemble EMD (EEMD), has been proposed in 2009 ([39]). EEMD performs an EMD over an ensemble of the signal under study plus Gaussian white noise. However, with EEMD new problems have been introduced: a residual noise is present in the signal reconstructed by EEMD and the mode mixing problem is again present in most applications to real data. To overcome this situation, another EMD-based algorithm, the complete EEMD with adaptive noise (CEEMDAN), has been presented in 2011 ([40]). CEEMDAN algorithm leads to an exact reconstruction of the original signal and a cleaner spectral separation of modes.

We propose in this paper to advance the knowledge of LSCI data by analyzing oscillations of laser speckle contrast image sequences through the use of the very recent CEEMDAN algorithm. Our goal is to answer the following questions: (i) from the temporal evolution of LSCI pixels what are the oscillations that can be extracted with the CEEMDAN algorithm? (ii) Are these oscillations similar when comparing time evolution of regions of interest (ROI) instead of time evolution of single pixels in laser speckle contrast images? (iii) Are the oscillations found in LSCI time series (temporal evolution of pixels or ROI) similar to the ones found in LDF time series recorded simultaneously to LSCI data? (iv) Finally, are the oscillation frequencies found with the CEEMDAN algorithm the same as the ones found with EMD and EEMD algorithms? In order to answer these four questions, we herein apply the CEEMDAN algorithm to LSCI and LDF data recorded simultaneously in healthy subjects. The latter data are also processed with EMD and EEMD algorithms.

\section{MATERIALS AND MEthods}

\section{A. Measurement procedure}

The measurement procedure that was performed for the data acquisition was carried out in accordance with the Declaration of Helsinki. All the subjects that were included in the work (nine Caucasian subjects) provided written, informed consent prior to participation. All the subjects were without known disease. They were placed supine in a quiet room with controlled temperature ([41]) and without any air movements ([42]). LSCI perfusion data were recorded on the right forearm dorsal face, in arbitrary laser speckle perfusion units $\left(\mathrm{APU}_{L S C I}\right)$. For this purpose, a PeriCam PSI System (Perimed, Sweden) having a laser wavelength of $785 \mathrm{~nm}$ and an exposure time of $6 \mathrm{~ms}$ was used. The distance between the laser head to skin was set at $15.5 \mathrm{~cm}$ ([43]) which gave images with a resolution around $0.44 \mathrm{~mm}$. LSCI images were recorded with a sampling frequency of $18 \mathrm{~Hz}$ during $13.8 \mathrm{~min}$ (15000 samples) and stored on a computer 
for an off-line analysis.

Moreover, for each subject, simultaneously to LSCI data acquisition, one LDF signal was recorded. For this purpose, a laser Doppler probe (model 455, Perimed, Sweden) was connected to a laser Doppler flowmeter having a $780 \mathrm{~nm}$ wavelength (PeriFlux System 5000, Perimed, Sweden) and positioned on the forearm that was imaged by the laser speckle contrast imager. LDF perfusion values were assessed in arbitrary laser Doppler perfusion units (APU $\left.\mathrm{ADF}_{L}\right)$ and recorded on a computer via an analog-to-digital converter (Biopac System) with a sampling frequency of $20 \mathrm{~Hz}$. A sub-sampling to $18 \mathrm{~Hz}$ was then performed.

On each LSCI recordings, one pixel was chosen arbitrarily in the first laser speckle contrast image of the images sequence, and followed with time. This led to one time series of 15000 samples for each of the nine subjects. The corresponding times series were processed with the CEEMDAN, EMD and EEMD algorithms as presented thereafter. Moreover, around each of the above-mentioned pixels, square ROI of size $3 \times 3$ pixels $^{2}\left(1.74 \mathrm{~mm}^{2}\right), 9 \times 9$ pixels $^{2}\left(15.68 \mathrm{~mm}^{2}\right), 15 \times 15$ pixels $^{2}\left(43.56 \mathrm{~mm}^{2}\right), 23 \times 23$ pixels $^{2}\left(102.41 \mathrm{~mm}^{2}\right), 31 \times 31$ pixels $^{2}\left(186.05 \mathrm{~mm}^{2}\right)$, and $61 \times 61$ pixels $^{2}\left(720.39 \mathrm{~mm}^{2}\right)$, were determined ([44], [45], [46], [47]). For each ROI, the mean of the pixel values (in $\mathrm{APU}_{L S C I}$ ) inside the ROI was computed and followed on each image of the sequence to obtain timeevolution signals. These new time-evolution signals were also processed with the CEEMDAN, EMD and EEMD algorithms.

\section{B. The empirical mode decomposition}

EMD consists in a local and fully data-driven separation of a signal in fast and slow oscillations and behaves as a dyadic filter bank ([48]). EMD relies on the decomposition of the signal under study into intrinsic mode functions (IMFs) or modes ([36]). A signal is considered as an IMF if it satisfies the two following conditions: (i) the number of extrema and the number of zero crossing must be equal or differ at most by one; (ii) the mean value of the upper and lower envelopes is zero everywhere.

For a discrete signal $x[n]$ the EMD algorithm is the following ([36])

1) identify all extrema of the signal $x[n]$

2) interpolate between minima (respectively maxima), ending up with some "envelope" $e_{\min }[n]$ (respectively $\left.e_{\max }[n]\right)$

3) compute the average $m[n]=\left(e_{\min }[n]+e_{\max }[n]\right) / 2$

4) extract the detail $d[n]=x[n]-m[n]$

5) iterate on the residual $m[n]$

The above algorithm has to be refined by a sifting process (an inner loop that iterates steps (1) to (4) upon the detail signal $d[n])$ until the detail signal $d[n]$ can be considered as zeromean from the stopping criterion ([49]). This leads to a detail considered as the effective IMF. Afterwards the corresponding residual $m[n]$ is computed and then step (5) applies. At the end we obtain

$$
x[n]=\sum_{k=1}^{K} I M F_{k}[n]+R[n],
$$

where $R[n]=m_{K}[n]$ is the residue that corresponds to the signal approximation at the lowest resolution, i.e. the trend and $I M F_{k}[n]$ is the $k$-th IMF ([36]). Thus, EMD performs a multi-scale decomposition. However, EMD has the drawback of leading to mode mixing: presence of oscillations of very disparate amplitude in a mode, or presence of very similar oscillations in different modes ([39]).

\section{The ensemble empirical mode decomposition}

EEMD has been proposed to overcome mode mixing in EMD. Thus EEMD is based on EMD where the signal processed is an ensemble constituted by the original signal and Gaussian white noise ([39]). The EEMD algorithm for a discrete signal $x[n]$ is the following

1) compute $x^{i}[n]=x[n]+w^{i}[n]$, where $w^{i}[n]$ with $i=1, \ldots, I$ are different realizations of white Gaussian noise

$2)$ decompose each $x^{i}[n](i=1, \ldots, I)$ with EMD. The corresponding modes $I M F_{k}^{i}[n]$ are obtained, where $k=$ $1, \ldots, K$ indicates the modes

3) compute the modes $\overline{I M F}_{k}$ of the EEMD method by averaging the $I M F_{k}^{i}$ as: $\overline{I M F}_{k}[n]=\frac{1}{I} \sum_{i=1}^{I} I M F_{k}^{i}[n]$

$\overline{I M F}_{k}$ does not necessarily satisfy the conditions required to be an IMF.

EEMD has the advantage, over EMD, of solving the mode mixing problem. However, it introduces other drawbacks: when reconstructing the signal after the application of EEMD, residual noise is obtained ([40]). Moreover, processing the same original signal several times with EEMD can produce different number of modes for each application ([40]).

\section{The complete ensemble empirical mode decomposition} with adaptive noise method

The CEEMDAN method has been proposed to provide an exact recontruction of the original signal and to obtain a better spectral separation of the modes ([40]). Moreover, it decreases the computational cost ([40]). CEEMDAN is based on EEMD. For a signal $x[n]$, the CEEMDAN algorithm is the following ([40])

1) compute $I$ realizations of $x[n]+\varepsilon_{0} w^{i}[n]$ where $w^{i}[n]$ with $i=1, \ldots, I$ are different realizations of white Gaussian noise and $\varepsilon_{0}$ is the noise standard deviation

2) decompose the above $I$ signals by EMD to obtain their first modes

3) compute the first mode $\widetilde{I M F_{1}}$ of the CEEMDAN method as

$$
\widetilde{I M F_{1}}[n]=\frac{1}{I} \sum_{i=1}^{I} I M F_{1}^{i}[n]=\overline{I M F}_{1}[n]
$$

4) calculate the first residue as

$$
r_{1}[n]=x[n]-\widetilde{I M F_{1}}[n]
$$


5) by defining $E_{j}(\cdot)$ as the operator that produces the $j$-th mode with the EMD algorithm, decompose realizations $r_{1}[n]+\varepsilon_{1} E_{1}\left(w^{i}[n]\right), i=1, \ldots, I$, until their first EMD mode. $\varepsilon_{k}(k=1$ for this step) allows to select the signal to noise ratio (SNR) at each stage. Then, define the second mode as

$$
\widetilde{I M F_{2}}[n]=\frac{1}{I} \sum_{i=1}^{I} E_{1}\left(r_{1}[n]+\varepsilon_{1} E_{1}\left(w^{i}[n]\right)\right)
$$

6) for $k=2, \ldots, K$ compute the $k$-th residue as

$$
r_{k}[n]=r_{(k-1)}[n]-\widetilde{I M F_{k}}[n]
$$

7) decompose realizations $r_{k}[n]+\varepsilon_{k} E_{k}\left(w^{i}[n]\right), i=$ $1, \ldots, I$, until their first EMD mode and define the $(k+1)$-th mode as

$$
\widetilde{I M F_{k+1}}[n]=\frac{1}{I} \sum_{i=1}^{I} E_{1}\left(r_{k}[n]+\varepsilon_{k} E_{k}\left(w^{i}[n]\right)\right)
$$

8) go to step 6 for next $k$

Steps 6 to 8 are done till the residue cannot be decomposed (when it does not contain at least two extrema). The final residue can be written as

$$
R[n]=x[n]-\sum_{k=1}^{K} \widetilde{I M F_{k}},
$$

where $K$ is the number total of modes. The CEEMDAN method has the advantage, over EEMD, of leading to a complete decomposition and leads to a numerically negligible error: the original signal $x[n]$ can be written as

$$
x[n]=\sum_{k=1}^{K} \widetilde{I M F_{k}}[n]+R[n] .
$$

Moreover, it gives a better spectral separation of the modes, needs a lower number of sifting iterations and thus is costcomputationaly interesting ([40]). In the CEEMDAN algorithm a particular noise is added at each stage of the decomposition. A unique residue is calculated to obtain each mode.

\section{RESULTS AND DISCUSSION}

To have a fair comparison between CEEMDAN and EEMD, the exact same set of values for the ensemble size and the noise amplitude have been used in the two algorithms. We used 500 realizations. Furthermore, we fixed the same SNR $\varepsilon_{k}$ value for all stages. Ten values for $\varepsilon_{k}$ have been tested: 0.1 to 4.6 by step of 0.5 . Moreover, for CEEMDAN, EMD and EEMD, the stopping criterion for sifting (the stopping criterion determines the number of sifting steps to produce an IMF) was chosen as described by Rilling et al. ([50]). This stopping criterion is based on two thresholds $\theta_{1}$ and $\theta_{2}$ to guarantee globally small fluctuations in the mean, while taking into account locally large excursions ([50]). The mode amplitude - defined as $\alpha(t)=\left(e_{\max }(t)-e_{\min }(t)\right) / 2$ - and the evaluation function - defined as $\sigma(t)=|m(t) / \alpha(t)|$ are introduced so that sifting is iterated until $\sigma(t)<\theta_{1}$ for some prescribed fraction $(1-\alpha)$ of the total duration, while $\sigma(t)<\theta_{2}$ for the remaining fraction ([50]). In our work, we set $\theta_{1}=0.05, \theta_{2}=0.5$ and $\alpha=0.05$, as recommended by Rilling et al. ([50]). The maximum number of sifting iterations allowed was set to 5000. Finally, based on the work by Rilling et al. ([50]), we have chosen a cubic spline interpolation. Indeed, Rilling et al. report that other types of interpolation (linear or polynomial) tend to increase the required number of sifting iterations and to "over-decompose" signals by spreading out their components over adjacent modes ([50]).

The average number of modes given by the CEEMDAN algorithm for LSCI and LDF data and for all the SNR $\varepsilon_{k}$ values tested are shown in Table I. From this table we observe that the average number of modes obtained with the CEEMDAN algorithm for LSCI data is between 14.7 and 16.0. For LDF it is between 14.7 and 15.7. Moreover, this number of modes does not vary much when the LSCI ROI sizes increase. Furthermore, for a given SNR $\varepsilon_{k}$ value, the average number of modes obtained with the CEEMDAN algorithm for LSCI data is close to the average one obtained for LDF signals. This is true whatever the LSCI ROI size. Moreover, we note that the number of modes shows a low increasing trend when the SNR $\varepsilon_{k}$ increases. This is true for LSCI data and LDF signals.

On each IMF obtained with the CEEMDAN algorithm, we determined the local extrema. From the latter, the average frequency of the oscillations has been determined. The results for LSCI data (single pixel time series and ROI times series) and LDF signals are presented in Tables II, and III, for each value of the SNR $\varepsilon_{k}$ tested. $I \widetilde{M} F_{1}$ to $I \widetilde{M} F_{3}$ are not shown as they correspond to frequencies higher than $2 \mathrm{~Hz}$ and therefore cannot correspond to physiological activities. Moreover, for clarity reasons in Tables, the lowest frequencies shown are the ones of $\widetilde{I M F_{13}}$. From these results we observe that

- for a given IMF, the average oscillation frequencies show small variations when the ROI size of LSCI data increases. This is true for all the SNR $\varepsilon_{k}$ values

- for a given IMF, the oscillation frequencies increase when the SNR $\varepsilon_{k}$ value increases. This is true for LSCI and LDF data. Thus, with LSCI data, for $\varepsilon_{k}=0.1$ and for a ROI size of $3 \times 3$ pixels $^{2}$, the average frequency oscillation for $\mathrm{IMF}_{4}$ is $1.431 \pm 0.043 \mathrm{~Hz}$ whereas it is of $2.899 \pm 0.032 \mathrm{~Hz}$ for $\varepsilon_{k}=4.6$

- for a given SNR $\varepsilon_{k}$ value the average oscillation frequencies obtained for LSCI data are close to the ones obtained for LDF signals

We also have processed the LSCI and LDF data with EMD and EEMD algorithms. As for the CEEMDAN results, we determined the local extrema of each IMF, from which the average frequency of the oscillations has been determined. The results are presented in Table IV for EMD and in Tables V and VI for EEMD. For EMD and EEMD, only $I M F_{4}$ to $I M F_{10}$ or $I M F_{11}$ are shown as some data had only 10 or $11 \mathrm{IMF}$. From EMD results (see Table IV) we observe that

- for a given IMF (LSCI data), the average oscillation frequencies obtained with a ROI of $1 \times 1$ pixels $^{2}$ is 
higher than the ones obtained with the other ROI sizes. This may be due to the fact that LSCI single pixels in time have statistical properties similar to those of white noises ([47]). For larger ROI sizes on LSCI data, the signal-to-noise ratio increases

- for ROI sizes higher than $1 \times 1$ pixels $^{2}$ on LSCI data and for a given IMF, the average oscillation frequencies show small variations when the ROI size on LSCI data increases

- for a given IMF, the average oscillation frequencies obtained with LSCI data is more than two times larger than the ones obtained with LDF data

- the average oscillation frequency obtained for $\mathrm{IMF}_{n}$ with LDF signals is close to the one obtained for $\mathrm{IMF}_{n+2}$ with LSCI data $(6 \leq n \leq 8)$

From EEMD results (see Tables V and VI) we observe that

- for a given IMF and for all SNR $\varepsilon_{k}$ values, for a ROI size higher than $1 \times 1$ pixels $^{2}$ in LSCI data, the average oscillation frequencies show small variations when the ROI size increases

- for a given IMF and for SNR $\varepsilon_{k}$ values higher than 2.1, the oscillation frequencies do not show large variations when the SNR $\varepsilon_{k}$ value increases. This is true for LSCI and LDF data. Thus, with LSCI data, for $\varepsilon_{k}=2.6$ and for a ROI size of $3 \times 3$ pixels $^{2}$, the average frequency oscillation for $\mathrm{IMF}_{4}$ is $1.106 \pm 0.031 \mathrm{~Hz}$ whereas it is of $1.108 \pm 0.040 \mathrm{~Hz}$ for $\varepsilon_{k}=4.6$

- for a SNR $\varepsilon_{k}$ value lower than 2.1, the average oscillation frequencies obtained for LDF data increases with $\mathrm{SNR} \varepsilon_{k}$ for a given IMF

- for a SNR $\varepsilon_{k}$ value larger than 2.1, the average oscillation frequencies obtained for LSCI data (ROI sizes higher than $1 \times 1$ pixels $^{2}$ ) are close to the ones obtained for LDF signals

A comparison of the average oscillation frequencies given by the CEEMDAN, EMD and EEMD algorithms shows that

- for LSCI data, for a given SNR $\varepsilon_{k}$ value in CEEMDAN and EEMD and algorithms, a given IMF and ROI size, the average oscillation frequency given by the CEEMDAN algorithm is higher than the one given by the EMD and EEMD algorithms

- for LDF signals, for a given SNR $\varepsilon_{k}$ value in CEEMDAN and EEMD algorithms and a given IMF, the average oscillation frequency given by the CEEMDAN algorithm is higher than the one given by the EMD and EEMD algorithms

- for LSCI data (ROI sizes higher than $1 \times 1$ pixels $^{2}$ ) and a given IMF, the average oscillation frequencies given by the EMD algorithm are in the same range as the ones given by the EEMD algorithm, whatever the SNR $\varepsilon_{k}$ value

- for LDF signals, the average oscillation frequencies given by the EMD algorithm for $\mathrm{IMF}_{k}$ are close to the ones given by the EEMD algorithm for $\mathrm{IMF}_{k+1}$ when the SNR $\varepsilon_{k}$ value is set to 0.6. However, for EEMD, the standard deviations are lower than the ones given by the EMD algorithm
All these results raise the question of the choice of the SNR $\varepsilon_{k}$ value in CEEMDAN and EEMD algorithms. It has been reported that, for CEEMDAN and EEMD, the added noise level and number of realizations can be adjusted depending on the application ([51]). Wu and Huang suggested to use small amplitude values for data dominated by high-frequency signals, and vice versa ([39]). However, from our results, we observe that this may be not an easy task. Using different values of the SNR $\varepsilon_{k}$, the information extracted from oscillations present in the data can be different.

From works based on wavelets, it has been reported that LDF signals contain six main frequencies in the frequency interval $0.0095-2.0 \mathrm{~Hz}$ ([52]) and each of these frequencies reflects a physiological process: $[0.6$ - 2.0] $\mathrm{Hz}$ (heartbeat), [0.145-0.6] Hz (respiratory activity), [0.052 - 0.145] $\mathrm{Hz}$ (intrinsic myogenic activity of vascular smooth muscle), [0.021 - 0.052] Hz (neurogenic (sympathetic) activity of the vessel vall), [0.0095 - 0.021] Hz (NO-dependent endothelial activity), [0.005 - 0.0095] Hz (non-NO-dependent endothelial activity) ([53]).

The oscillation frequencies obtained with the EMD algorithm on LDF signals can be classified in these intervals: $0.502 \mathrm{~Hz}$ and $0.228 \mathrm{~Hz}$ in the interval of the respiratory activity; $0.109 \mathrm{~Hz}$ and $0.056 \mathrm{~Hz}$ in the interval of the intrinsic myogenic activity of vascular smooth muscle; $0.027 \mathrm{~Hz}$ in the interval of the sympathetic activity; $0.014 \mathrm{~Hz}$ in the interval of the NO-dependent activity and $0.007 \mathrm{~Hz}$ in the interval of the non-NO-dependent endothelial activity. The presence of two oscillations in the frequency range corresponding to the respiratory and intrinsic myogenic activities remains to be studied.

EMD is an adaptive method: it is based and derived from the data. By opposition, Fourier analyses or time scale wavelet decompositions require a predetermined basis: sinusoidal functions for Fourier analyses and mother wavelets (e.g., Morlet, Daubechies, or many others) for wavelet decompositions. LDF signals have extensively been studied through wavelets (see, e.g., [54], [55], [56], [57]). However, from the best of our knowledge, only a few EMD-based analyses have been performed on such signals ([58], [59], [60]). Moreover, as far as we are concerned, no EMD study has been conducted on LSCI data. Our work is therefore the first one to present an EMD-based analysis of LSCI data and to compare the results with those obtained with LDF signals recorded simultaneously.

It is recognized that EMD and EEMD have the drawback of requiring long computation time (see, e.g., [61]). However, a recent study reported that the time complexity of EMD and EEMD is equivalent to the one of the Fourier transform ([62]).

In our work, a cubic spline interpolation has been used as the envelope fitting method, for CEEMDAN, EEMD and EMD. Other methods could be tested (see, e.g., [63] and references inside, as well as [64]). 


\section{CONCLusions}

EMD is a non linear and non stationary time domain decomposition method that has the advantage of relying on an adaptive and data-driven algorithm. When applied on a signal, IMFs are extracted and each one represents a narrow band-frequency-amplitude modulation that often corresponds to a specific physical phenomenon. However, EMD has some drawbacks that EEMD tried to overcome. EEMD first generates an ensemble of data sets computed by adding different realizations of a white noise with finite amplitude to the original time series. The EMD algorithm is then applied to each data series of the ensemble. The IMFs are computed by averaging the respective components in each realization over the ensemble. However, when reconstructing the signal after the application of EEMD, residual noise is obtained. Moreover, processing the same original signal several times with EEMD can produce different number of modes for each application. The CEEMDAN algorithm has very recently been proposed to face out these problems.

In our work, images from the recent LSCI technique are processed to extract oscillations that may be present in the time domain. For this purpose, several image sequences recorded in healthy subjects have been analyzed with the CEEMDAN algorithm. Moreover, the results have been compared with the ones obtained from EMD and EEMD algorithms.

Furthermore, LDF signals recorded simultaneously to the LSCI data have also been studied and processed with the CEEMDAN, EMD and EEMD algorithms. The average frequencies of the oscillations obtained with these three methods have been compared and analyzed with the ones given by LSCI data.

The results show that values of the average frequencies given by the CEEMDAN algorithm depend on the SNR used in the computation but, for a given SNR, the average frequencies found for LSCI data are close to the ones obtained for LDF signals. By opposition, EEMD leads to average frequencies that show low variations when the SNR is higher than a threshold. Moreover, for a given SNR and a given IMF, the values of the oscillation frequencies given by the CEEMDAN algorithm are higher than the ones given by EEMD and EMD algorithms, both for LSCI and LDF data. Finally, the oscillation frequencies obtained with EMD on LDF signals are found in intervals previously reported in works based on wavelet analyses. The CEEMDAN algorithm has the advantage of achieving a complete decomposition with no error in the reconstruction but our study suggests that further work is needed to gain knowledge in the adjustment of the added noise level.

\section{REFERENCES}

[1] K. Winter, L.H. Metz, J.P. Kuska, and B. Frerich, "Characteristic quantities of microvascular structures in CLSM volume datasets," IEEE Trans. Med. Imaging, vol. 26, pp. 1103-1114, 2007.

[2] D. Gil, O. Rodriguez-Leor, P. Radeva, and J. Mauri, "Myocardial perfusion characterization from contrast angiography spectral distribution," IEEE Trans. Med. Imaging, vol. 27, pp. 641-649, 2008.

[3] Friman, O., Hindennach, M., Kühnel, C., Peitgen, H.O., "Multiple hypothesis template tracking of small 3D vessel structures", Med. Image Anal., vol. 14, pp. 160-171, 2010.
[4] E.M. Kohner, "The effect of diabetic control on diabetic retinopathy", Eye (Lond), vol. 7, pp. 309-311, 1993.

[5] F. Jung, G. Pindur, P. Ohlmann, G. Spitzer, R. Sternitzky, R.P. Franke, B. Leithäuser, S. Wolf, and J.W. Park, "Microcirculation in hypertensive patients," Biorheology, vol. 50, pp. 241-255, 2013.

[6] A.L. Herrick, "Vascular function in systemic sclerosis", Curr. Opin. Rheumatol., vol. 12, pp. 527-533, 2000.

[7] F.M. Wigley, "Vascular disease in scleroderma", Clin. Rev. Allergy Immunol., vol. 36, pp. 150-175, 2009.

[8] M. Roustit and J.L. Cracowski, "Non-invasive assessment of skin microvascular function in humans: an insight into methods", Microcirculation, vol. 19, pp. 47-64, 2012.

[9] A. Humeau-Heurtier, E. Guerreschi, P. Abraham, and G. Mahé, "Relevance of laser Doppler and laser speckle techniques for assessing vascular function: state of the art and future trends", IEEE Trans. Biomed. Eng., vol. 60, pp. 659-666, 2013.

[10] M.D. Stern, "In vivo evaluation of microcirculation by coherent light scattering", Nature, vol. 254, pp. 56-58, 1975.

[11] Fredriksson, I., Fors, C., Johansson, J., "Laser Dopper flowmetry - a theoretical framework. Department of Biomedical Engineering, Linköping University (2007), www.imt.liu.se/bit/ldf/ldfmain.html

[12] R. Bonner, and R. Nossal, "Model for laser Doppler measurements of blood flow in tissue", Appl. Opt., vol. 20, pp. 2097-2107, 1981.

[13] M. Roustit, S. Blaise, C. Millet, and J.L. Cracowski, "Reproducibility and methodological issues of skin post-occlusive and thermal hyperemia assessed by single-point laser Doppler flowmetry", Microvasc. Res., vol. 79, pp. 102-108, 2010.

[14] M. Roustit, C. Millet, S. Blaise, B. Dufournet, and J.L. Cracowski, "Excellent reproducibility of laser speckle contrast imaging to assess skin microvascular reactivity”, Microvasc. Res., vol. 80, pp. 505-511, 2010.

[15] Essex, T.J., Byrne, P.O., "A laser Doppler scanner for imaging blood flow in skin", J. Biomed. Eng., vol. 13, pp. 189-194, 1991.

[16] K. Wardell, A. Jakobsson, and G.E. Nilsson, , "Laser Doppler perfusion imaging by dynamic light scattering", IEEE Trans. Biomed. Eng., vol. 40, pp. 309-316, 1993.

[17] A. Serov, W. Steenbergen, and F. de Mul, "Laser Doppler perfusion imaging with a complimentary metal oxide semiconductor image sensor", Opt. Lett., vol. 27, pp. 300-302, 2002.

[18] A. Serov and T. Lasser, "High-speed laser Doppler perfusion imaging using an integrating CMOS image sensor", Opt. Express, vol. 13, pp. 6416-6428, 2005.

[19] A. Serov, B. Steinacher, and T. Lasser, "Full-field laser Doppler perfusion imaging and monitoring with an intelligent CMOS camera", Opt. Express, vol. 13, pp. 3681-3689, 2005.

[20] Draijer, M., Hondebrink, E., van Leeuwen, T., Steenbergen, W., "Twente Optical Perfusion Camera: system overview and performance for video rate laser Doppler perfusion imaging", Opt. Express, vol. 17, pp. 32113225, 2009.

[21] M. Leutenegger, E. Martin-Williams, P. Harbi, T. Thacher, W. Raffoul, M. André, A. Lopez, P. Lasser, and T. Lasser, "Real-time full field laser Doppler imaging", Biomed. Opt. Express, vol. 2, pp. 1470-1477, 2011.

[22] He, D., Nguyen, H.C., Hayes-Gill, B.R., Zhu, Y., Crowe, J.A., Clough, G.F., Gill, C.A., Morgan, S.P., " $64 \times 64$ pixel smart sensor array for laser Doppler blood flow imaging”, Opt. Lett., vol. 37, pp. 3060-3062, 2012.

[23] He, D., Nguyen, H.C., Hayes-Gill, B.R., Zhu, Y., Crowe, J.A., Gill, C., Clough, G.F., Morgan, S.P., "Laser doppler blood flow imaging using a CMOS imaging sensor with on-chip signal processing", Sensors, vol. 13, pp. 12632-12647, 2013.

[24] Harbi, P., Thacher, T., "Body mapping of human cutaneous microcirculatory perfusion using a real-time laser Doppler imager", Diab. Vasc. Dis. Res., vol. 10, pp. 187-190, 2013.

[25] A. Ponticorvo, and A.K. Dunn, "How to build a Laser Speckle Contrast Imaging (LSCI) system to monitor blood flow", J. Vis. Exp., vol. 45. pii: 2004. doi: 10.3791/2004, 2010.

[26] L.M. Richards, S.M. Kazmi, J.L. Davis, K.E. Olin, and A.K. Dunn, "Low-cost laser speckle contrast imaging of blood flow using a webcam", Biomed. Opt. Express, vol. 4, pp. 2269-2283, 2013.

[27] M. Le Thinh, J.S. Paul, H. Al-Nashash, A. Tan, A.R. Luft, F.S. Sheu, and S.H. Ong, "New insights into image processing of cortical blood flow monitors using laser speckle imaging", IEEE Trans. Med. Imaging, vol. 26, pp. 833-842, 2007

[28] W.J. Tom, A. Ponticorvo, and A.K. Dunn, "Efficient processing of laser speckle contrast images", IEEE Trans. Med. Imaging, vol. 27, pp. 1728$1738,2008$.

[29] J. Wang, Y. Wang, B. Li, D. Feng, J. Lu, Q. Luo, and P. Li, "Dualwavelength laser speckle imaging to simultaneously access blood flow, 
blood volume, and oxygenation using a color CCD camera", Opt. Lett., vol. 38, pp. 3690-3692, 2013.

[30] S.M. Narayan, and J.M. Smith, "Spectral analysis of periodic fluctuations in electrocardiographic repolarization", IEEE Trans. Biomed. Eng., vol. 46, 203-212, 1999.

[31] Bricq, S., Mahé, G., Rousseau, D., Humeau-Heurtier, A., ChapeauBlondeau, F., Varela, J.R., Abraham, P., "Assessing spatial resolution versus sensitivity from laser speckle contrast imaging: application to frequency analysis", Med. Biol. Eng. Comput, vol. 50, pp. 1017-1023, 2012.

[32] G. Soloperto, F. Conversano, A. Greco, E. Casciaro, R. Franchini, and S. Casciaro, "Advanced spectral analyses for real-time automatic echographic tissue-typing of simulated tumor masses at different compression stages", IEEE Trans. Ultrason. Ferroelectr. Freq. Control., vol. 59, pp. 2692-2701, 2012.

[33] R. Maniewski, P. Leger, P. Lewandowski, A. Liebert, P. Bendayan, H. Boccalon, L. Bajorski, and K.O. Möller, "Spectral analysis of laserDoppler perfusion signal measured during thermal test", Technol. Health Care, vol. 7, pp. 163-169, 1999.

[34] R. Weidenhagen, A. Wichmann, H.G. Koebe, L. Lauterjung, H. Fürst, and K. Messmer, "Analysis of laser Doppler flux motion in man: comparison of autoregressive modelling and fast Fourier transformation", Int J. Microcirc. Clin. Exp., vol. 16, pp. 64-73, 1996.

[35] A. Stefanovska, M. Bracic, and H.D. Kvernmo, "Wavelet analysis of oscillations in the peripheral blood circulation measured by laser Doppler technique", IEEE Trans. Biomed. Eng., vol. 46, pp. 1230-1239, 1999.

[36] N.E. Huang, Z. Shen, S.R. Long, M.C. Wu, H.H. Shih, Q. Zheng, N.C. Yen, C.C. Tung, and H.H. Liu, "The empirical mode decomposition and the Hilbert spectrum for nonlinear and non-stationary time series analysis", Proc. R. Soc. London A, vol. 454, pp. 903-995, 1998.

[37] P. Flandrin, P. Goncalves, and G. Rilling, "Detrending and denoising with Empirical Mode Decompositions", EUSIPCO-04, pp. 1581-1584, Wien (A), 2004.

[38] T. Pereira, P. Vaz, T. Oliveira, I. Santos, H.C. Pereira, V. Almeida, C. Correia, and J. Cardoso, "Empirical mode decomposition for self-mixing Doppler signals of hemodynamic optical probes", Physiol. Meas., vol. 34, pp. 377-390, 2013

[39] Z. Wu and N.E. Huang, "Ensemble empirical mode decomposition: a noise-assisted data analysis method", Adv. Adapt. Data Anal., vol. 1, pp. $1-41,2009$

[40] M.E. Torres, M.A. Colominas, G. Schlotthauer, and P. Flandrin, "A complete ensemble empirical mode decomposition with adaptive noise", IEEE International Conference on Acoustics, Speech and Signal Processing (ICASSP), pp. 4144-4147, 2011.

[41] P. Abraham, M. Bourgeau, M. Camo, A. Humeau-Heurtier, S. Durand, P. Rousseau, G. Mahe, "Effect of skin temperature on skin endothelial function assessment," Microvasc. Res., vol. 88, pp. 56-60, 2013.

[42] G. Mahe, S. Durand, A. Humeau, G. Leftheriotis, P. Rousseau, and P. Abraham, "Air movements interfere with laser speckle contrast imaging recordings", Lasers Med. Sci., vol. 27, pp. 1073-1076, 2011.

[43] G. Mahe, F. Haj-Yassin, P. Rousseau, A. Humeau, S. Durand, G. Leftheriotis, and P. Abraham, "Distance between laser head and skin does not influence skin blood flow values recorded by laser speckle imaging", Microvasc. Res., vol. 82, pp. 439-442, 2011.

[44] P. Rousseau, G. Mahé, F. Haj-Yassin, S. Durand, A. Humeau, G. Leftheriotis, and P. Abraham, "Increasing the "region of interest" and "time of interest", both reduce the variability of blood flow measurements using laser speckle contrast imaging", Microvasc. Res., vol. 82, pp. 88-91, 2011.

[45] A. Humeau-Heurtier, G. Mahé, S. Durand, D. Henrion, and P. Abraham, "Laser speckle contrast imaging: multifractal analysis of data recorded in healthy subjects", Med. Phys., vol. 39, pp. 5849-5856, 2012.
[46] A. Humeau-Heurtier, P. Abraham, and G. Mahe, "Linguistic analysis of laser speckle contrast images recorded at rest and during biological zero: comparison with laser Doppler flowmetry data", IEEE Trans. Med. Imaging, vol. 32, pp. 2311-2321, 2013.

[47] A. Humeau-Heurtier, G. Mahé, S. Durand, and P. Abraham, "Multiscale entropy study of medical laser speckle contrast images", IEEE Trans. Biomed. Eng., vol. 60, pp. 872-879, 2013.

[48] Flandrin, P., Rilling, G., Goncalves, P., "Empirical Mode Decomposition as a Filter Bank", IEEE Sig. Proc. Lett., vol. 11, pp. 112-114, 2004.

[49] N.E. Huang, M.C. Wu, S.R. Long, S.S.P. Shen, W. Qu, P. Gloersen, and K.L Fan, "A confidence limit for the empirical mode decomposition and Hilbert spectral analysis,", Proc. R. Soc. London A, vol. 459, pp. 2317-2345, 2003.

[50] G. Rilling, P. Flandrin,, and P. Conçalvès, "On Empirical Mode Decomposition and its Algorithms", IEEE-EURASIP Workshop on Nonlinear Signal and Image Processing NSIP-03, 2003, Grado (I), 2003.

[51] Colominas, M.A., Schlotthauer, G., Torres, M.E., Flandrin, P., "Noiseassisted EMD methods in action", Adv. Adapt. Data Anal., vol. 4, pp. 1250025-1-1250025-11, 2012.

[52] P. Kvandal, S.A. Landsverk, A. Bernjak, A. Stefanovska, H.D. Kvernmo, and K.A. Kirkeboen, "Low-frequency oscillations of the laser Doppler perfusion signal in human skin", Microvasc. Res., vol. 72, pp. 120-127, 2006.

[53] A. Bernjak, P.B. Clarkson, P.V. McClintock, A. Stefanovska, "Lowfrequency blood flow oscillations in congestive heart failure and after beta1-blockade treatment," Microvasc. Res., vol. 76, pp. 224-232, 2008.

[54] A. Humeau, J.L. Saumet, and J.P. L'Huillier, "Use of wavelets to accurately determine parameters of laser Doppler reactive hyperemia", Microvasc. Res., vol. 60, pp. 141-148, 2000.

[55] A. Humeau, A. Koitka, J.L. Saumet, and J.P. L'Huillier, "Wavelet denoising of laser Doppler reactive hyperemia signals to diagnose peripheral arterial occlusive diseases", IEEE Trans. Biomed. Eng., vol. 49, pp. 13691371, 2002.

[56] A. Bernjak, G.A. Deitrick, W.A. Bauman, A. Stefanovska, and J. Tuckman, "Basal sympathetic activity to the microcirculation in tetraplegic man revealed by wavelet transform of laser Doppler flowmetry," $M i$ crovasc. Res., vol. 81, pp. 313-318, 2011.

[57] Y.K. Jan, B. Lee, F. Liao, and R.D. Foreman, "Local cooling reduces skin ischemia under surface pressure in rats: an assessment by wavelet analysis of laser Doppler blood flow oscillations", Physiol. Meas., vol. 33, pp. 1733-1745, 2012.

[58] S. Assous, A. Humeau, and J.P. L'huillier, "Empirical mode decomposition applied to laser Doppler flowmetry signals : diagnosis approach," Conf. Proc. IEEE Eng. Med. Biol. Soc., vol. 2, pp. 1232-1235, 2005.

[59] A. Humeau, W. Trzepizur, D. Rousseau, F. Chapeau-Blondeau, and P. Abraham, "Localization of transient signal high-values in laser Doppler flowmetry signals with an empirical mode decomposition", Med. Phys., vol. 36, pp. 18-21, 2009.

[60] F. Liao and Y.K. Jan, "Enhanced phase synchronization of blood flow oscillations between heated and adjacent non-heated sacral skin", Med. Biol. Eng. Comput., vol. 50, pp. 1059-1070, 2012.

[61] M.H. Lee, K.K. Shyu, P.L. Lee, C.M. Huang, and Y.J. Chiu, "Hardware implementation of EMD using DSP and FPGA for online signal processing”, IEEE Trans. Industr. Electron., vol. 58, pp. 2473-2481, 2011.

[62] Y.H.Wang, C.H. Yeh, H.W.V. Young, K. Hu, and M.T. Lo, "On the computational complexity of the empirical mode decomposition algorithm", Physica A, vol. 400, pp. 159-167, 2014.

[63] W. Zhu, H. Zhao, D. Xiang, and X. Chen, "A flattest constrained envelope approach for empirical mode decomposition," Plos One, vol. 8, e61739, 2013.

[64] K. Zeng and M.X. He, "A simple boundary process technique for empirical mode decomposition", IEEE Int. Symp. Geoscience and Remote Sensing, IGARSS'04, vol. 6, pp. 4258-4261, 2004. 
TABLE I

AVERAGE NUMBER OF MODES GIVEN BY THE CEEMDAN ALGORITHM FOR LSCI AND LDF DATA RECORDED ON THE FOREARM SKIN OF 9 HEALTHY SUBJECTS. RESULTS FOR DIFFERENT VALUES OF THE SNR $\varepsilon_{k}$ ARE PRESENTED. LSCI $x \times x$ WHERE $x$ IS A NUMBER REFERS TO RESULTS OBTAINED WITH LSCI DATA AVERAGED ON A ROI OF SIZE $x \times x$ PIXELS $^{2}$.

\begin{tabular}{ccccccccc}
\hline $\begin{array}{c}\varepsilon_{k} \\
\text { value }\end{array}$ & $1 \times 1$ & $3 \times 3$ & $9 \times 9$ & $15 \times 15$ & $23 \times 23$ & $31 \times 31$ & $61 \times 61$ & \\
\hline 0.1 & 15 & 14.8 & 14.8 & 14.7 & 14.7 & 14.7 & 14.7 & 14.7 \\
\hline 0.6 & 15.1 & 15.0 & 15.0 & 14.9 & 14.9 & 14.9 & 14.9 & 14.8 \\
\hline 1.1 & 15.4 & 15.2 & 15.1 & 15.0 & 14.9 & 15.0 & 15.2 & 15.1 \\
\hline 1.6 & 15.3 & 15.2 & 15.2 & 15.3 & 14.9 & 15.3 & 15.1 & 15.1 \\
\hline 2.1 & 15.6 & 15.8 & 15.3 & 15.6 & 15.7 & 15.1 & 15.2 & 15.3 \\
\hline 2.6 & 15.4 & 15.4 & 15.2 & 15.6 & 15.3 & 15.1 & 15.4 & 15.4 \\
\hline 3.1 & 15.7 & 15.7 & 15.4 & 15.1 & 15.6 & 15.9 & 15.4 & 15.7 \\
\hline 3.6 & 15.9 & 15.7 & 15.7 & 15.7 & 15.4 & 15.6 & 15.7 & 15.6 \\
\hline 4.1 & 16.0 & 15.9 & 15.4 & 15.9 & 15.7 & 15.8 & 15.7 & 15.4 \\
\hline 4.6 & 16.0 & 15.8 & 15.8 & 15.6 & 16.0 & 15.6 & 15.7 & 15.7 \\
\hline & & & & & & & &
\end{tabular}

TABLE II

AVERAGE FREQUENCY (IN HZ) OF THE OSCILLATIONS COMPUTED FROM $I M F_{4}$ TO $\widetilde{I M F_{13}}$ OBTAINED WITH THE CEEMDAN ALGORITHM FOR LSCI AND LDF DATA RECORDED ON THE FOREARM SKIN OF 9 HEALTHY SUBJECTS. RESULTS FOR DIFFERENT VALUES OF THE SNR $\varepsilon_{k}$ ARE PRESENTED. LSCI $x \times x$ WHERE $x$ IS A NUMBER REFERS TO RESULTS OBTAINED WITH LSCI DATA AVERAGED ON A ROI OF SIZE $x \times x$ PIXELS ${ }^{2}$. THE VALUES IN PARENTHESES CORRESPOND TO THE STANDARD DEVIATIONS.

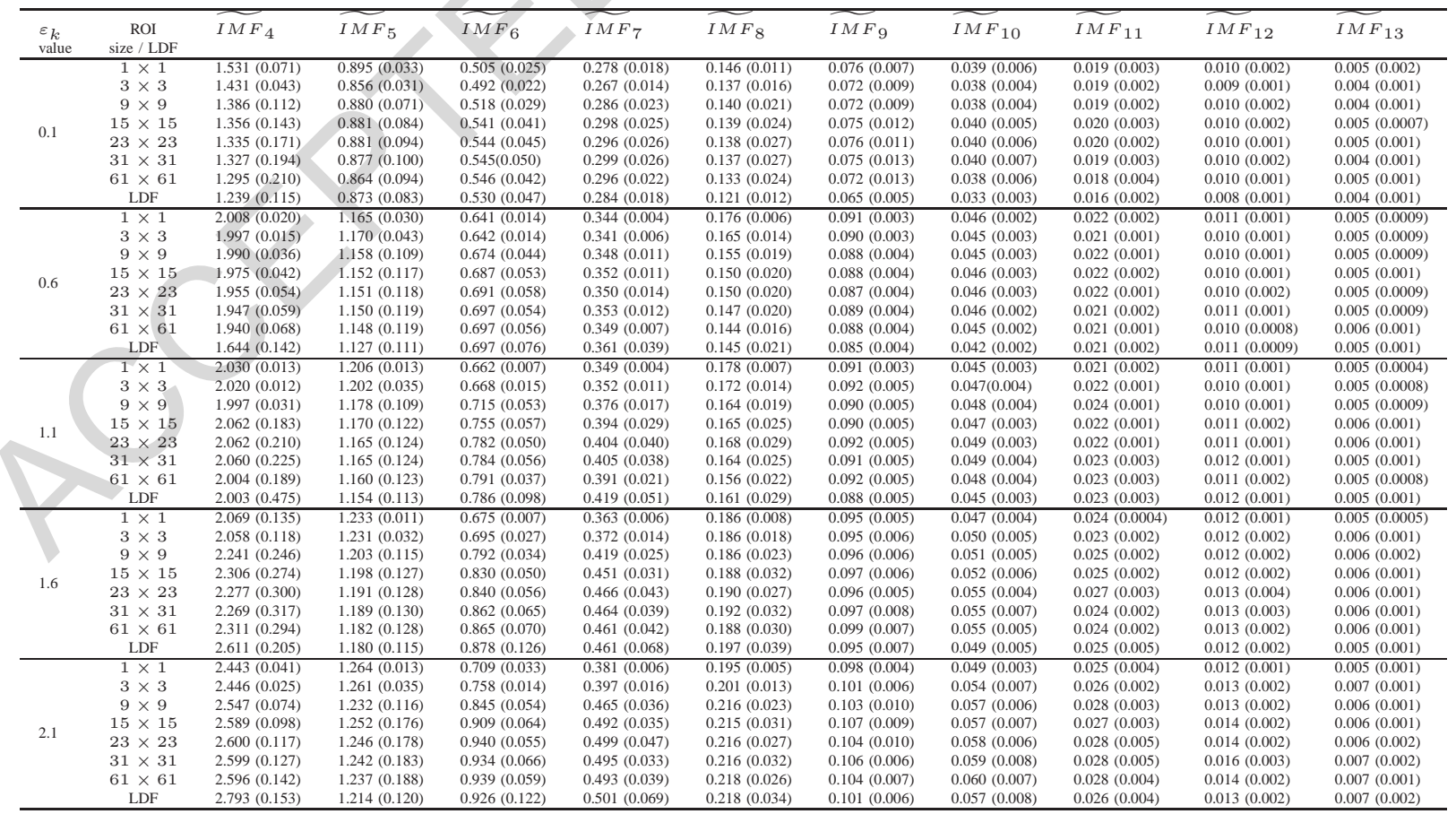


TABLE III

AVERAGE FREQUENCY (IN HZ) OF THE OSCILLATIONS COMPUTED FROM $I M F_{4}$ TO $\widetilde{I M F}{ }_{13}$ OBTAINED WITH THE CEEMDAN ALGORITHM FOR LSCI AND LDF DATA RECORDED ON THE FOREARM SKIN OF 9 HEALTHY SUBJECTS. RESULTS FOR DIFFERENT VALUES OF THE SNR $\varepsilon_{k}$ ARE PRESENTED. LSCI $x \times x$ WHERE $x$ IS A NUMBER REFERS TO RESULTS OBTAINED WITH LSCI DATA AVERAGED ON A ROI OF SIZE $x \times x$ PIXELS ${ }^{2}$. THE VALUES IN PARENTHESES CORRESPOND TO THE STANDARD DEVIATIONS.

\begin{tabular}{|c|c|c|c|c|c|c|c|c|c|c|c|}
\hline $\begin{array}{c}\varepsilon_{k} \\
\text { value }\end{array}$ & $\begin{array}{c}\text { ROI } \\
\text { size / LDF }\end{array}$ & $I M F_{4}$ & $I M F_{5}$ & $I M F_{6}$ & $I M F_{7}$ & $I M F_{8}$ & $I M F_{9}$ & $I M F 10$ & $I M F_{11}$ & $I M F_{12}$ & $I M F_{13}$ \\
\hline \multirow{8}{*}{2.6} & $1 \times 1$ & $2.558(0.058)$ & $1.297(0.019)$ & $0.759(0.012)$ & $0.407(0.008)$ & $0.214(0.011)$ & $0.108(0.007)$ & $0.053(0.008)$ & $0.028(0.002)$ & $0.014(0.003)$ & $0.006(0.001)$ \\
\hline & $3 \times 3$ & $2.559(0.041)$ & $1.297(0.031)$ & $0.790(0.019)$ & $0.428(0.024)$ & $0.217(0.016)$ & $0.111(0.006)$ & $0.058(0.007)$ & $0.029(0.003)$ & $0.013(0.002)$ & $0.007(0.002)$ \\
\hline & $9 \times 9$ & $2.665(0.081)$ & $1.304(0.142)$ & $0.932(0.039)$ & $0.499(0.039)$ & $0.235(0.016)$ & $0.111(0.009)$ & $0.062(0.004)$ & $0.030(0.003)$ & $0.015(0.002)$ & $0.007(0.002)$ \\
\hline & $15 \times 15$ & $2.706(0.111)$ & $1.302(0.162)$ & $0.966(0.053)$ & $0.526(0.024)$ & $0.243(0.029)$ & $0.114(0.010)$ & $0.061(0.007)$ & $0.030(0.005)$ & $0.016(0.003)$ & $0.008(0.002)$ \\
\hline & $23 \times 23$ & $2.716(0.118)$ & $1.284(0.197)$ & $0.979(0.028)$ & $0.530(0.028)$ & $0.236(0.029)$ & $0.111(0.006)$ & $0.059(0.010)$ & $0.034(0.005)$ & $0.015(0.003)$ & $0.007(0.002)$ \\
\hline & $31 \times 31$ & $2.717(0.123)$ & $1.277(0.198)$ & $0.981(0.028)$ & $0.527(0.040)$ & $0.239(0.030)$ & $0.116(0.011)$ & $0.062(0.007)$ & $0.031(0.004)$ & $0.014(0.002)$ & $0.008(0.001)$ \\
\hline & $61 \times 61$ & $2.707(0.131)$ & $1.269(0.202)$ & $0.990(0.026)$ & $0.531(0.031)$ & $0.235(0.024)$ & $0.113(0.010)$ & $0.064(0.005)$ & $0.032(0.003)$ & $0.016(0.003)$ & $0.007(0.001)$ \\
\hline & LDF & $2.917(0.143)$ & $1.303(0.165)$ & $0.958(0.101)$ & $0.527(0.062)$ & $0.237(0.030)$ & $0.113(0.011)$ & $0.058(0.005)$ & $0.028(0.004)$ & $0.015(0.003)$ & $0.008(0.002)$ \\
\hline \multirow{8}{*}{3.1} & $1 \times 1$ & $2.644(0.054)$ & $1.351(0.088)$ & $0.797(0.020)$ & $0.432(0.017)$ & $0.227(0.009)$ & $0.120(0.006)$ & $0.057(0.004)$ & $0.032(0.003)$ & $0.015(0.002)$ & $0.007(0.001)$ \\
\hline & $3 \times 3$ & $2.665(0.035)$ & $1.400(0.130)$ & $0.816(0.010)$ & $0.457(0.011)$ & $0.236(0.017)$ & $0.120(0.010)$ & $0.063(0.005)$ & $0.030(0.003)$ & $0.015(0.002)$ & $0.008(0.001)$ \\
\hline & $9 \times 9$ & $2.779(0.076)$ & $1.382(0.183)$ & $0.981(0.020)$ & $0.516(0.032)$ & $0.249(0.018)$ & $0.117(0.012)$ & $0.068(0.007)$ & $0.034(0.003)$ & $0.018(0.003)$ & $0.009(0.002)$ \\
\hline & $15 \times 15$ & $2.816(0.087)$ & $1.374(0.189)$ & $1.003(0.026)$ & $0.545(0.027)$ & $0.258(0.019)$ & $0.120(0.011)$ & $0.068(0.007)$ & $0.034(0.005)$ & $0.017(0.003)$ & $0.010(0.002)$ \\
\hline & $23 \times 23$ & $2.818(0.100)$ & $1.327(0.176)$ & $1.005(0.021)$ & $0.539(0.031)$ & $0.260(0.020)$ & $0.123(0.012)$ & $0.069(0.005)$ & $0.035(0.003)$ & $0.016(0.004)$ & $0.008(0.001)$ \\
\hline & $31 \times 31$ & $2.832(0.121)$ & $1.318(0.176)$ & $1.003(0.028)$ & $0.544(0.028)$ & $0.256(0.024)$ & $0.121(0.013)$ & $0.070(0.007)$ & $0.033(0.004)$ & $0.017(0.002)$ & $0.009(0.001)$ \\
\hline & $61 \times 61$ & $2.825(0.132)$ & & & $0.554(0.036)$ & & & $0.068(0.006)$ & $0.034(0.005)$ & $0.017(0.002)$ & $0.009(0.001)$ \\
\hline & LDF & $2.994(0.103)$ & $1.402(0.192)$ & $0.982(0.095)$ & $0.548(0.059)$ & $0.258(0.025)$ & $0.116(0.011)$ & $0.064(0.005)$ & $0.031(0.003)$ & $0.016(0.002)$ & $0.009(0.001)$ \\
\hline \multirow{8}{*}{3.6} & $1 \times 1$ & $2.740(0.048)$ & $1.594(0.034)$ & $0.825(0.037)$ & $0.457(0.007)$ & $0.242(0.009)$ & $0.128(0.008)$ & $0.063(0.005)$ & $0.032(0.003)$ & $0.018(0.004)$ & $0.008(0.001)$ \\
\hline & $3 \times 3$ & $2.753(0.038)$ & $1.542(0.116)$ & $0.885(0.055)$ & $0.485(0.023)$ & $0.254(0.012)$ & $0.123(0.013)$ & $0.070(0.008)$ & $0.033(0.004)$ & $0.018(0.003)$ & $0.008(0.001)$ \\
\hline & $9 \times 9$ & $2.860(0.067)$ & $1.476(0.208)$ & $0.999(0.022)$ & $0.548(0.026)$ & $0.265(0.020)$ & 0.126 & $71(0.005)$ & $0.036(0.004)$ & $0.018(0.002)$ & $0.008(0.001)$ \\
\hline & $15 \times 15$ & $2.895(0.099)$ & $1.469(0.209)$ & & $0.556(0.028)$ & & & & & & $0.008(0.003)$ \\
\hline & $23 \times 23$ & $2.906(0.092)$ & $1.446(0.214)$ & $1.021(0.022)$ & $0.567(0.028)$ & $0.269(0.016)$ & $0.130(0.009)$ & $0.071(0.007)$ & $0.034(0.003)$ & $0.017(0.002)$ & $0.008(0.002)$ \\
\hline & $31 \times 31$ & $2.908(0.098)$ & $1.446(0.222)$ & $1.021(0.023)$ & $0.561(0.033)$ & $0.263(0.019)$ & $0.129(0.011)$ & $0.069(0.006)$ & $0.034(0.005)$ & $0.019(0.002)$ & $0.008(0.001)$ \\
\hline & $61 \times 61$ & $2.896(0.102)$ & $1.408(0.218)$ & $1.027(0.018)$ & $0.559(0.029)$ & $0.272(0.019)$ & $0.128(0.012)$ & $0.073(0.005)$ & $0.036(0.005)$ & $0.018(0.003)$ & $0.008(0.002)$ \\
\hline & $\hat{\mathrm{LDF}}$ & $3.054(0.087)$ & $1.485(0.15$ & $1.008(0.078)$ & $0.566(0.046)$ & $0.271(0.022)$ & $0.126(0.014)$ & $0.067(0.003)$ & $0.034(0.004)$ & $0.017(0.003)$ & $0.009(0.001)$ \\
\hline \multirow{8}{*}{4.1} & $1 \times 1$ & $2.830(0.040)$ & & $0.873(0$. & & & & & & & $0.009(0.002)$ \\
\hline & $3 \times 3$ & $2.828(0.027)$ & $1.632(0$ & $0.942(0$ & 0.507 & 5) & 0.134 & & $0.035(0.005)$ & $0.019(0.001)$ & $0.008(0.0009)$ \\
\hline & $9 \times 9$ & $2.919(0.0$ & & & & & & & & & $0.009(0.001)$ \\
\hline & $15 \times 15$ & $2.967(0.074)$ & $1.532(0.198)$ & $1.026(0.018)$ & $0.579(0.016)$ & $0.280(0.014)$ & $0.132(0.010)$ & $0.074(0.005)$ & $0.038(0.004)$ & $0.017(0.004)$ & $0.009(0.001)$ \\
\hline & $23 \times 23$ & $2.974(0.087)$ & $1.496(0.224)$ & $1.031(0.016)$ & $0.578(0.025)$ & $0.273(0.014)$ & $0.137(0.010)$ & $0.076(0.006)$ & $0.039(0.005)$ & $0.018(0.003)$ & $0.009(0.002)$ \\
\hline & $31 \times 31$ & $2.980(0.08$ & & & & & & & & & $0.009(0.002)$ \\
\hline & $61 \times 61$ & $2.962(0.0$ & 1.467( & & & & & & & & \\
\hline & LDF & $3.100(0.072)$ & $1.605(0.1$ & $1.030(0.04$ & 0.578 ( C & $0.276(0$. & 0.134 & & & $0.018(0.003)$ & $0.009(0.001)$ \\
\hline \multirow{8}{*}{4.6} & $1 \times 1$ & $2.889(0.040)$ & $1.686(0.023)$ & $0.942(0.022)$ & $0.507(0.014)$ & $0.263(0.009)$ & $0.138(0.007)$ & $0.065(0.005)$ & $0.033(0.004)$ & $0.018(0.003)$ & $0.009(0.001)$ \\
\hline & $3 \times 3$ & $2.899(0.032)$ & $1.683(0.021)$ & $0.973(0.016)$ & $0.532(0.021)$ & $0.276(0.012)$ & $0.135(0.010)$ & $0.075(0.003)$ & $0.036(0.002)$ & $0.018(0.003)$ & $0.010(0.001)$ \\
\hline & & $2.997(0.05$ & & $1.028(0.015)$ & & & & & & & $0.009(0.001)$ \\
\hline & $15 \times 15$ & & & & & & & & & & \\
\hline & $23 \times 23$ & $20(0.0$ & 1.6 & & & 0. & $0.144(0$ & & $88(0.004)$ & $9(0.002)$ & $0.010(0.001)$ \\
\hline & $31 \times 31$ & $3.025(0.082)$ & $1.564(0.198)$ & $1.046(0.020)$ & $0.591(0.018)$ & $0.294(0.015)$ & $0.142(0.009)$ & $0.075(0.005)$ & $0.039(0.004)$ & $0.017(0.002)$ & $0.010(0.001)$ \\
\hline & $61 \times 61$ & $3.012(0.082)$ & $1.513(0.231)$ & $1.047(0.014)$ & $0.579(0.026)$ & $0.288(0.017)$ & $0.142(0.008)$ & $0.078(0.004)$ & $0.038(0.002)$ & $0.018(0.002)$ & $0.009(0.002)$ \\
\hline & $\mathrm{LDF}$ & $3136(0057)$ & $1674(0.078)$ & $1038(0.041)$ & $0577(0.034)$ & $0203(0.019)$ & $0138(0010)$ & $0.073(0.005)$ & $0.038(0.003)$ & $0.019(0.002)$ & $0.010<0.002$ \\
\hline
\end{tabular}

TABLE IV

AVER AGE FREQUENCY (IN HZ) OF THE OSCILLATIONS COMPUTED FROM $I M F_{4}$ TO $I M F_{10}$ OBTAINED WITH THE EMD ALGORITHM FOR LSCI AND LDF DATA RECORDED ON THE FOREARM SKIN OF 9 HEALTHY SUBJECTS. LSCI $x \times x$ WHERE $x$ IS A NUMBER REFERS TO RESULTS OBTAINED WITH LSCI DATA AVERAGED ON A ROI OF SIZE $x \times x$ PIXELS $^{2}$. THE VALUES IN PARENTHESES CORRESPOND TO THE STANDARD DEVIATIONS.

\begin{tabular}{|c|c|c|c|c|c|c|c|}
\hline $\begin{array}{c}\text { ROI } \\
\text { size / LDF }\end{array}$ & $I M F_{4}$ & $I M F_{5}$ & $I M F_{6}$ & $I M F_{7}$ & $I M F_{8}$ & $I M F_{9}$ & $I M F_{10}$ \\
\hline $1 \times 1$ & $1.391(0.095)$ & $0.809(0.090)$ & $0.457(0.071)$ & $0.249(0.044)$ & $0.130(0.025)$ & $0.070(0.017)$ & $0.035(0.008)$ \\
\hline $3 \times 3$ & $1.174(0.089)$ & $0.662(0.071)$ & $0.353(0.054)$ & $0.179(0.042)$ & $0.092(0.024)$ & $0.048(0.013)$ & $0.025(0.007)$ \\
\hline $9 \times 9$ & $1.084(0.076)$ & $0.634(0.067)$ & $0.336(0.062)$ & $0.164(0.052)$ & $0.086(0.030)$ & $0.044(0.018)$ & $0.023(0.012)$ \\
\hline $15 \times 15$ & $1.096(0.124)$ & $0.670(0.127)$ & $0.374(0.103)$ & $0.188(0.086)$ & $0.101(0.056)$ & $0.053(0.029)$ & $0.028(0.018)$ \\
\hline $23 \times 23$ & $1.077(0.141)$ & $0.657(0.109)$ & $0.357(0.086)$ & $0.178(0.071)$ & $0.097(0.042)$ & $0.052(0.025)$ & $0.027(0.015)$ \\
\hline $31 \times 31$ & $1.044(0.176)$ & $0.616(0.160)$ & $0.348(0.158)$ & $0.176(0.104)$ & $0.097(0.062)$ & $0.053(0.040)$ & $0.027(0.024)$ \\
\hline $61 \times 61$ & $1.021(0.105)$ & $0.601(0.106)$ & $0.333(0.110)$ & $0.174(0.095)$ & $0.095(0.058)$ & $0.047(0.029)$ & $0.024(0.017)$ \\
\hline LDF & $0.502(0.114)$ & $0.228(0.085)$ & $0.109(0.043)$ & $0.056(0.023)$ & $0.027(0.013)$ & $0.014(0.006)$ & $0.007(0.003)$ \\
\hline
\end{tabular}


TABLE V

AVERAGE FREQUENCY (IN Hz) OF THE OSCILLATIONS COMPUTED FROM $\overline{I M F}_{4}$ TO $\overline{I M F}_{13}$ OBTAINED WITH THE EEMD ALGORITHM FOR LSCI AND

LDF DATA RECORDED ON THE FOREARM SKIN OF 9 HEALTHY SUBJECTS. RESULTS FOR DIFFERENT VALUES OF THE SNR $\varepsilon_{k}$ ARE PRESENTED. LSCI $x \times x$ WHERE $x$ IS A NUMBER REFERS TO RESULTS OBTAINED WITH LSCI DATA AVERAGED ON A ROI OF SIZE $x \times x$ PIXELS ${ }^{2}$. THE VALUES IN PARENTHESES CORRESPOND TO THE STANDARD DEVIATIONS.

\begin{tabular}{|c|c|c|c|c|c|c|c|c|c|}
\hline $\begin{array}{l}\varepsilon_{k} \\
\text { value }\end{array}$ & $\begin{array}{c}\text { ROI } \\
\text { size / LDF }\end{array}$ & $\overline{I M F}_{4}$ & $\overline{I M F_{5}}$ & $\overline{I M F}_{6}$ & $\overline{I M F_{7}}$ & $\overline{I M F} 8$ & $\overline{I M F_{9}}$ & $\overline{I M F} 10$ & $\overline{I M F}_{11}$ \\
\hline \multirow{8}{*}{0.1} & $1 \times 1$ & $1.374(0.046)$ & $0.794(0.048)$ & $0.447(0.030)$ & $0.242(0.022)$ & $0.127(0.016)$ & $0.066(0.010)$ & $0.034(0.007)$ & $0.017(0.004)$ \\
\hline & $3 \times 3$ & $1.224(0.064)$ & $0.689(0.051)$ & $0.370(0.037)$ & $0.188(0.034)$ & $0.099(0.016)$ & $0.051(0.010)$ & $0.027(0.006)$ & $0.012(0.003)$ \\
\hline & $9 \times 9$ & $1.137(0.097)$ & $0.642(0.058)$ & $0.349(0.052)$ & $0.171(0.053)$ & $0.090(0.027)$ & $0.050(0.018)$ & $0.025(0.010)$ & $0.013(0.006)$ \\
\hline & $15 \times 15$ & $1.122(0.108)$ & $0.635(0.073)$ & $0.344(0.072)$ & $0.167(0.058)$ & $0.090(0.030)$ & $0.049(0.021)$ & $0.025(0.010)$ & $0.012(0.007)$ \\
\hline & $23 \times 23$ & $1.124(0.134)$ & $0.641(0.110)$ & $0.352(0.089)$ & $0.172(0.074)$ & $0.095(0.040)$ & $0.050(0.024)$ & $0.026(0.014)$ & $0.013(0.008)$ \\
\hline & $31 \times 31$ & $1.120(0.137)$ & $0.640(0.116)$ & $0.350(0.094)$ & $0.172(0.075)$ & $0.094(0.040)$ & $0.050(0.025)$ & $0.027(0.015)$ & $0.014(0.008)$ \\
\hline & $61 \times 61$ & $1.098(0.118)$ & $0.615(0.105)$ & $0.335(0.084)$ & $0.160(0.069)$ & $0.090(0.040)$ & $0.047(0.023)$ & $0.024(0.014)$ & $0.013(0.008)$ \\
\hline & LDF & $0.657(0.033)$ & $0.330(0.030)$ & $0.137(0.029)$ & $0.072(0.010)$ & $0.035(0.004)$ & $0.018(0.004)$ & $0.008(0.002)$ & $0.004(0.0009)$ \\
\hline \multirow{8}{*}{0.6} & $1 \times 1$ & $1.271(0.026)$ & $0.722(0.035)$ & $0.395(0.018)$ & $0.211(0.016)$ & $0.111(0.011)$ & $0.058(0.008)$ & $0.028(0.005)$ & $0.014(0.002)$ \\
\hline & $3 \times 3$ & $1.185(0.055)$ & $0.663(0.037)$ & $0.354(0.027)$ & $0.178(0.029)$ & $0.095(0.011)$ & $0.048(0.007)$ & $0.024(0.004)$ & $0.011(0.003)$ \\
\hline & $9 \times 9$ & $1.148(0.112)$ & $0.630(0.040)$ & $0.335(0.039)$ & $0.160(0.042)$ & $0.086(0.019)$ & $0.046(0.012)$ & $0.023(0.006)$ & $0.011(0.004)$ \\
\hline & $15 \times 15$ & $1.146(0.115)$ & $0.628(0.046)$ & $0.330(0.050)$ & $0.156(0.044)$ & $0.087(0.021)$ & $0.046(0.015)$ & $0.023(0.008)$ & \\
\hline & $23 \times 23$ & $1.147(0.116)$ & $0.625(0.056)$ & $0.328(0.049)$ & $0.155(0.047)$ & $0.086(0.023)$ & $0.046(0.016)$ & $0.023(0.009)$ & $0.012(0.006)$ \\
\hline & $31 \times 31$ & $1.147(0.116)$ & $0.623(0.058)$ & $0.326(0.049)$ & $0.154(0.047)$ & $0.086(0.023)$ & $0.046(0.017)$ & $0.023(0.009)$ & $0.012(0.005)$ \\
\hline & $61 \times 61$ & $1.143(0.115)$ & $0.608(0.067)$ & $0.319(0.049)$ & $0.148(0.046)$ & $0.083(0.022)$ & $0.045(0.017)$ & $0.022(0.009)$ & $0.011(0.005)$ \\
\hline & LDF & $1.054(0.099)$ & $0.502(0.025)$ & $0.227(0.030)$ & $0.107(0.010)$ & $0.056(0.007)$ & $0.028(0.003)$ & $0.014(0.001)$ & $0.008(0.001)$ \\
\hline \multirow{8}{*}{1.1} & $1 \times 1$ & $1.191(0.027)$ & $0.656(0.029)$ & $0.352(0.011)$ & $0.183(0.010)$ & $0.096(0.006)$ & $0.049(0.004)$ & $0.025(0.002)$ & $0.012(0.001)$ \\
\hline & $3 \times 3$ & $1.154(0.048)$ & $0.632(0.025)$ & $0.333(0.015)$ & $0.164(0.021)$ & $0.090(0.007)$ & $0.045(0.005)$ & $0.022(0.003)$ & $0.010(0.002)$ \\
\hline & $9 \times 9$ & $1.136(0.113)$ & $0.626(0.038)$ & $0.325(0.022)$ & $0.148(0.028)$ & $0.083(0.010)$ & $0.044(0.007)$ & $0.022(0.004)$ & $0.011(0.003)$ \\
\hline & $15 \times 15$ & $1.139(0.118)$ & $0.631(0.037)$ & $0.318(0.025)$ & $0.146(0.031)$ & $0.082(0.012)$ & $0.043(0.008)$ & $0.022(0.004)$ & $0.010(0.003)$ \\
\hline & $23 \times 23$ & $1.141(0.119)$ & $0.634(0.049)$ & $0.319(0.026)$ & $0.146(0.034)$ & $0.082(0.012)$ & $0.043(0.009)$ & $0.022(0.006)$ & $0.010(0.003)$ \\
\hline & $31 \times 31$ & $1.141(0.118)$ & $0.633(0.046)$ & & $0.145(0.033)$ & $0.083(0.012)$ & $0.043(0.010)$ & $0.021(0.006)$ & $0.010(0.003)$ \\
\hline & $61 \times 61$ & $1.139(0.118)$ & $0.622(0.040)$ & $0.311(0.026)$ & $0.140(0.031)$ & $0.081(0.013)$ & $0.042(0.009)$ & $0.020(0.006)$ & $0.010(0.003)$ \\
\hline & LDF & $1.102(0.114)$ & $0.561(0.024)$ & $0.270(0.016)$ & $0.116(0.012)$ & $0.065(0.004)$ & $0.031(0.003)$ & $0.015(0.002)$ & $0.008(0.001)$ \\
\hline \multirow{8}{*}{1.6} & $1 \times 1$ & $1.122(0.026)$ & $0.627(0.022)$ & $0.334(0.011)$ & $0.172(0.010)$ & $0.090(0.006)$ & $0.045(0.004)$ & $0.023(0.001)$ & $0.012(0.001)$ \\
\hline & $3 \times 3$ & $1.123(0.045)$ & $0.615(0.0$ & $0.323(0.009)$ & $0.157(0.016)$ & $0.086(0.004)$ & $0.043(0.004)$ & $0.021(0.002)$ & $0.010(0.001)$ \\
\hline & $9 \times 9$ & $1.132(0.113)$ & $3(0$. & $20(0$. & & 0.083 (0 & & & $0.010(0.002)$ \\
\hline & $15 \times 15$ & $1.137(0.119)$ & $0.630(0.049)$ & $0.312(0.015)$ & $0.141(0.025)$ & $0.082(0.007)$ & $0.042(0.005)$ & $0.021(0.003)$ & $0.010(0.002)$ \\
\hline & $23 \times 23$ & $1.139(0.120)$ & $0.633(0.060)$ & $0.314(0.014)$ & $0.138(0.023)$ & $0.081(0.008)$ & $0.042(0.006)$ & $0.021(0.004)$ & $0.010(0.003)$ \\
\hline & $31 \times 31$ & $1.139(0.119)$ & $0.632(0.065)$ & $0.314(0.014)$ & $0.139(0.023)$ & $0.081(0.0$ & $0.042(0.005)$ & $0.020(0.004)$ & $0.010(0.002)$ \\
\hline & $61 \times 61$ & $1.138(0.119)$ & $0.628(0.0$ & $0.308(0.016)$ & $0.136(0.021)$ & $0.081(0.008)$ & $0.041(0.006)$ & $0.019(0.004)$ & $0.010(0.002)$ \\
\hline & LDF & $1.109(0.112)$ & $0.590(0.040)$ & $0.287(0.013)$ & $0.122(0.015)$ & $0.070(0.003)$ & $0.033(0.002)$ & $0.017(0.002)$ & $0.008(0.001)$ \\
\hline \multirow{8}{*}{2.1} & $1 \times 1$ & $1.100(0.012)$ & $0.614(0.013)$ & $0.323(0.010)$ & $0.165(0.008)$ & $0.087(0.004)$ & $0.042(0.003)$ & $0.021(0.001)$ & $0.011(0.001)$ \\
\hline & $3 \times 3$ & $1.117(0.0$ & $0.608(0.6$ & $0.320(0.0$ & $0.155(0.0$ & $0.084(0$. & $0.042(0.6$ & $0.021(0.002)$ & $0.010(0.001)$ \\
\hline & $9 \times 9$ & $1.131(0.111)$ & $0.617(0.043)$ & $0.315(0.010)$ & $0.140(0.017)$ & $0.082(0.006)$ & $0.041(0.004)$ & $0.020(0.002)$ & $0.010(0.002)$ \\
\hline & $15 \times 15$ & $1.136(0.118)$ & $0.632(0.057)$ & $0.312(0.010)$ & $0.137(0.019)$ & $0.081(0.006)$ & $0.041(0.004)$ & $0.020(0.003)$ & $0.010(0.002)$ \\
\hline & $23 \times 23$ & $1.138(0.120)$ & $0.632(0.0$ & $0.313(0.009)$ & $0.136(0.018)$ & $0.082(0.005)$ & $0.042(0.004)$ & $0.020(0.002)$ & $0.010(0.002)$ \\
\hline & $31 \times 31$ & $1.137(0.11$ & $1(0$. & $0.311(0.010)$ & 0.1 . & $0.082(0.006)$ & $0.041(0.004)$ & $0.020(0.003)$ & $0.010(0.002)$ \\
\hline & $61 \times 61$ & $1.138(0.119)$ & $0.636(0.069)$ & $0.309(0.011)$ & $0.133(0.016)$ & $0.082(0.005)$ & $0.041(0.004)$ & $0.019(0.003)$ & $0.010(0.002)$ \\
\hline & LDF & $1.111(0.111)$ & $0.607(0.048)$ & $0.294(0.010)$ & $0.123(0.016)$ & $0.074(0.003)$ & $0.035(0.001)$ & $0.017(0.002)$ & $0.009(0.001)$ \\
\hline
\end{tabular}

TABLE VI

AVERAGE FREQUENCY (IN HZ) OF THE OSCILLATIONS COMPUTED FROM $\overline{I M F}_{4}$ TO $\overline{I M F}_{13}$ OBTAINED WITH THE EEMD ALGORITHM FOR LSCI AND

LDF DATA RECORDED ON THE FOREARM SKIN OF 9 HEALTHY SUBJECTS. RESULTS FOR DIFFERENT VALUES OF THE SNR $\varepsilon_{k}$ ARE PRESENTED. LSCI $x \times x$ WHERE $x$ IS A NUMBER REFERS TO RESULTS OBTAINED WITH LSCI DATA AVERAGED ON A ROI OF SIZE $x \times x$ PIXELS ${ }^{2}$. THE VALUES IN PARENTHESES CORRESPOND TO THE STANDARD DEVIATIONS.

\begin{tabular}{|c|c|c|c|c|c|c|c|c|c|}
\hline $\begin{array}{c}\varepsilon_{k} \\
\text { value }\end{array}$ & $\begin{array}{c}\text { ROI } \\
\text { size / LDF }\end{array}$ & $\overline{I M F}_{4}$ & $\overline{I M F}_{5}$ & $\overline{I M F} 6$ & $\overline{I M F}_{7}$ & $\overline{I M F} 8$ & $\overline{I M F} 9$ & $\overline{I M F}_{10}$ & $\overline{I M F}_{11}$ \\
\hline \multirow{8}{*}{2.6} & $1 \times 1$ & $1.093(0.011)$ & $0.608(0.012)$ & $0.322(0.007)$ & $0.163(0.007)$ & $0.085(0.005)$ & $0.042(0.004)$ & $0.021(0.001)$ & $0.011(0.001)$ \\
\hline & $3 \times 3$ & $1.106(0.031)$ & $0.610(0.013)$ & $0.316(0.009)$ & $0.153(0.013)$ & $0.083(0.004)$ & $0.042(0.003)$ & $0.020(0.001)$ & $0.009(0.001)$ \\
\hline & $9 \times 9$ & $1.129(0.110)$ & $0.619(0.046)$ & $0.314(0.006)$ & $0.138(0.016)$ & $0.083(0.005)$ & $0.041(0.003)$ & $0.020(0.001)$ & $0.009(0.001)$ \\
\hline & $15 \times 15$ & $1.137(0.118)$ & $0.630(0.066)$ & $0.311(0.005)$ & $0.135(0.015)$ & $0.081(0.006)$ & $0.042(0.003)$ & $0.020(0.002)$ & $0.009(0.001)$ \\
\hline & $23 \times 23$ & $1.137(0.119)$ & $0.634(0.074)$ & $0.309(0.007)$ & $0.133(0.014)$ & $0.081(0.005)$ & $0.041(0.003)$ & $0.020(0.002)$ & $0.009(0.002)$ \\
\hline & $31 \times 31$ & $1.136(0.119)$ & $0.636(0.071)$ & $0.308(0.008)$ & $0.133(0.015)$ & $0.083(0.006)$ & $0.042(0.003)$ & $0.019(0.002)$ & $0.010(0.002)$ \\
\hline & $61 \times 61$ & $1.135(0.118)$ & $0.634(0.070)$ & $0.308(0.007)$ & $0.133(0.016)$ & $0.082(0.005)$ & $0.042(0.003)$ & $0.019(0.002)$ & $0.010(0.002)$ \\
\hline & LDF & $1.112(0.109)$ & $0.617(0.057)$ & $0.298(0.011)$ & $0.125(0.016)$ & $0.075(0.003)$ & $0.036(0.001)$ & $0.017(0.002)$ & $0.009(0.001)$ \\
\hline \multirow{8}{*}{3.1} & $1 \times 1$ & $1.094(0.010)$ & $0.606(0.007)$ & $0.320(0.006)$ & $0.163(0.007)$ & $0.086(0.004)$ & $0.042(0.004)$ & $0.020(0.0007)$ & $0.011(0.001)$ \\
\hline & $3 \times 3$ & $1.114(0.043)$ & $0.608(0.011)$ & $0.315(0.010)$ & $0.151(0.012)$ & $0.084(0.004)$ & $0.042(0.002)$ & $0.020(0.001)$ & $0.010(0.0008)$ \\
\hline & $9 \times 9$ & $1.129(0.112)$ & $0.616(0.043)$ & $0.309(0.006)$ & $0.137(0.015)$ & $0.082(0.006)$ & $0.041(0.002)$ & $0.020(0.001)$ & $0.009(0.001)$ \\
\hline & $15 \times 15$ & $1.135(0.117)$ & $0.630(0.067)$ & $0.306(0.007)$ & $0.135(0.015)$ & $0.082(0.006)$ & $0.041(0.003)$ & $0.020(0.002)$ & $0.009(0.0009)$ \\
\hline & $23 \times 23$ & $1.136(0.118)$ & $0.633(0.071)$ & $0.310(0.006)$ & $0.134(0.016)$ & $0.082(0.005)$ & $0.041(0.002)$ & $0.019(0.002)$ & $0.009(0.001)$ \\
\hline & $31 \times 31$ & $1.135(0.119)$ & $0.636(0.073)$ & $0.311(0.007)$ & $0.132(0.014)$ & $0.084(0.005)$ & $0.042(0.003)$ & $0.019(0.002)$ & $0.010(0.002)$ \\
\hline & $61 \times 61$ & $1.137(0.118)$ & $0.635(0.075)$ & $0.311(0.006)$ & $0.132(0.014)$ & $0.083(0.005)$ & $0.041(0.003)$ & $0.018(0.002)$ & $0.010(0.002)$ \\
\hline & LDF & $1.113(0.110)$ & $0.614(0.054)$ & $0.300(0.012)$ & $0.127(0.017)$ & $0.077(0.004)$ & $0.037(0.001)$ & $0.018(0.001)$ & $0.009(0.001)$ \\
\hline \multirow{8}{*}{3.6} & $1 \times 1$ & $1.094(0.010)$ & $0.607(0.007)$ & $0.320(0.007)$ & $0.162(0.008)$ & $0.084(0.004)$ & $0.040(0.003)$ & $0.020(0.001)$ & $0.010(0.0007)$ \\
\hline & $3 \times 3$ & $1.114(0.043)$ & $0.604(0.016)$ & $0.312(0.008)$ & $0.150(0.011)$ & $0.083(0.004)$ & $0.041(0.003)$ & $0.019(0.001)$ & $0.009(0.0008)$ \\
\hline & $9 \times 9$ & $1.129(0.111)$ & $0.616(0.050)$ & $0.312(0.006)$ & $0.138(0.015)$ & $0.083(0.006)$ & $0.041(0.003)$ & $0.020(0.0009)$ & $0.009(0.001)$ \\
\hline & $15 \times 15$ & $1.136(0.117)$ & $0.631(0.065)$ & $0.309(0.006)$ & $0.135(0.015)$ & $0.082(0.006)$ & $0.042(0.003)$ & $0.019(0.001)$ & $0.009(0.001)$ \\
\hline & $23 \times 23$ & $1.137(0.119)$ & $0.633(0.0$ & 0.311 ( & & $82(0.006)$ & $0.041(0.0$ & & $0.009(0.001)$ \\
\hline & $31 \times 31$ & & & & & & & & \\
\hline & $61 \times 61$ & $1.137(0.118)$ & $0.637(0.073)$ & $0.309(0.007)$ & $0.130(0.012)$ & 005) & $0.042(0.003)$ & $9(0.002)$ & $0.010(0.001)$ \\
\hline & LDF & $1.111(0.110)$ & $0.626(0.069)$ & $0.299(0.012)$ & $0.016)$ & $0.078(0.005)$ & $0.038(0.001)$ & $0.018(0.002)$ & $0.009(0.001)$ \\
\hline \multirow{8}{*}{4.1} & $1 \times 1$ & $1.089(0$. & $0.603(0$. & $0.318(0.1$ & & $0.085(0$ & $0.040(0.002)$ & $0.020(0.0009)$ & $0.010(0.0009)$ \\
\hline & $3 \times 3$ & $1.102(0.025)$ & $0.603(0$. & & & & $0.041(C$ & & $0.009(0.001)$ \\
\hline & $9 \times 9$ & $1.129(0.108)$ & $0.621(0$. & & & & & & $0.001)$ \\
\hline & $15 \times 15$ & $1.133(0.116)$ & 0.63 & & & & 12) & & $0.009(0.0009)$ \\
\hline & & $1.138(0.1$ & $0.632(0.0$ & 0.30 & $0.133(0$. & $0.082(0$. & $0.042(0.0$ & $0.019(0.001)$ & $0.009(0.001)$ \\
\hline & $31 \times 31$ & $1.138(0.119)$ & $0.635(0.07$ & $0.311(0.0$ & $0.132(0.0$ & $0.083(0.004)$ & $0.042(0.002)$ & $0.019(0.001)$ & $0.009(0.001)$ \\
\hline & $61 \times 61$ & $1.136(0.119)$ & $0.637(0.074)$ & 0700 & & $0.083(0.004)$ & $0.042(0.002)$ & $0.018(0.001)$ & $0.010(0.001)$ \\
\hline & LDF & & & & & & & & $0.009(0.001)$ \\
\hline \multirow{8}{*}{4.6} & $1 \times 1$ & & & & & & 4) & & $0.010(0.001)$ \\
\hline & $3 \times 3$ & $1.108(0.040)$ & 0.6 & 0.3 & $0.152(0$. & 0. & $0.042(0.002)$ & $0.019(0.0007)$ & $0.010(0.001)$ \\
\hline & $9 \times 9$ & $1.129(0.109)$ & $0.618(0.046)$ & $0.315(0.004)$ & $0.136(0.013)$ & $0.082(0.005)$ & $0.041(0.003)$ & $0.020(0.001)$ & $0.009(0.001)$ \\
\hline & $15 \times 15$ & $1.134(0.115)$ & $0.631(0.064)$ & $0.311(0.008)$ & $0.135(0.01$ & $0.082(0.006)$ & $0.042(0.003)$ & $0.019(0.0009)$ & $0.009(0.0008)$ \\
\hline & & & & & & & & & $0.009(0.0007)$ \\
\hline & & & & & & & & & \\
\hline & $61 \times 61$ & $1.136(0.116)$ & $0.639(0.073)$ & $0.310(0.008)$ & $0.131(0.012)$ & $0.083(0.004)$ & $0.042(0.002)$ & $0.018(0.002)$ & $0.009(0.001)$ \\
\hline & LDF & $1.115(0.111)$ & $0.631(0.068)$ & $0.304(0.011)$ & $0.128(0.017)$ & $0.080(0.005)$ & $0.038(0.001)$ & $0.018(0.001)$ & $0.010(0.001)$ \\
\hline
\end{tabular}

